\title{
Self-Forces on Electric and Magnetic Linear Sources in the Space-Time of a Cosmic String
}

\author{
E. R. Bezerra de Mello* and V. B. Bezerra ${ }^{\dagger}$ \\ Departamento de Física, UFPB, 58051-970, J.Pessoa,PB, \\ BRAZIL \\ C. Furtado and F. Moraes ${ }^{\diamond}$ \\ Departamento de Física, UFPE, 50670-901, Recife, PE, \\ BRAZIL
}

\begin{abstract}
In this paper we calculate the magnetic and electric self-forces, induced by the conical structure of a cosmic string space-time, on a long straight wire which presents either a constant current or a linear charge density. We also show how these self-forces are related by a Lorentz tranformation and, in this way, explain what two different inertial observers detect in their respective frames.
\end{abstract}

PACS numbers: 98.80.cq, 04.40.+c, 41.20.-q

*e-mail: cendfi50@brufpb.bitnet

†Present address:

${ }^{\diamond}$ Present address:

Blackett Laboratory

School of Natural Sciences

Imperial College

London SW7 2BZ

Institute for Advanced Study

UK

Princeton, NJ 08540

USA 
It is well known that the space-time produced by a thin, infinite, straight cosmic string, has no Newtonian potential [1] and cannot induce curvature (locally the curvature vanishes everywhere except at the source). However, there are some global non-trivial topological effects associated with this space-time which can be measured [2].

Recently, Linet [3] and Smith [4] have shown that a charged point particle placed in a static space-time, produced by an infinite straight cosmic string, becomes subjected to a finite repulsive electric self-force. This phenomenon is a consenquence of the distortion in the particle field caused by the lack of global flatness of this space-time.

The purpose of this paper is to investigate how the self-force phenomenon manisfests in the magnetic case; i.e., we study what happens either to an electric current or to a linear charge distribution in the conical space-time produced by a cosmic string. Furthermore, we show that the respective selfforces are related by a Lorentz transformation (a boost along the direction of the string). In order to analyse this problem we shall adopt, for simplicity only, the case where the magnetic and electric sources are both infinite straight wires parallel to the cosmic string, which we assume lies along the z-axis. Before we start our calculation, we add that the analysis presented here can also be applied to some elastic solids which present a specific kind of linear defects named disclinations [5]. As shown by Katanaev and Volovich [6], the change in the topology of the elastic medium produced by this linear defect is the same as the one produced by a cosmic string in Minkowski spacetime. This similarity goes beyond topology: for some applications both kinds of defects can be treated using the same geometrical approach; i.e., their surrounding space-time can be described by similar geometrical points of view. In this approach, the line element in the section $\mathrm{z}=\mathrm{t}=$ constant, is given by

$$
d \vec{\ell}_{(2)}^{2}=d r^{2}+r^{2} d \theta^{2}
$$


where $r \geq 0$ and $0 \leq \theta \leq 2 \pi \alpha$, being $\alpha=1 / p$ a parameter related to the linear mass density $\mu$ of the cosmic string by $\alpha=1-4 \mu G / c^{2}$. For an elastic solid $\alpha=1+\lambda / 2 \pi$, where $\lambda$ is the angle which defines the wedge [7] For the cosmic string, $\alpha$ is smaller than 1. For disclinations, however, it can also assume values greater than 1 , which correspond to an anti-conical space-time with negative curvature.

Before going on into the calculations let us say a few words concerning the motivation to study this problem. Certainly, the understanding of the interaction between currents and a topological defect, such as a cosmic string, can give us some informations on the behavior of a superconducting string [8] in the space-time of the defect. Also, at a low energy level, it may be useful in the study of electromagnetic properties of disclinated media $[5,9]$.

Now, after this brief introduction about our problem, let us first analyse the easiest case of a linear charge distribution parallel to the string.

The electrostatic potential energy for an arbitrary charge distribution is:

$$
U_{\text {Ele }}=1 / 2 \int d^{3} r \rho(\vec{r}) \Phi(\vec{r}),
$$

where $\rho(\vec{r})$ is the charge density and $\Phi(\vec{r})$ the scalar potential. Because we are interested in studying the problem of the electrostatic self-energy for a constant linear charge density parallel to the string, the Maxwell equation for the scalar potential

$$
\nabla^{2} \Phi(\vec{r})=-4 \pi \rho(\vec{r})
$$

reduces to a two-dimensional one. In this case, we can write the electrostatic potential energy per unit length as

$$
\frac{U_{\text {Ele }}}{\ell}=1 / 2 \iint d^{2} r d^{2} r^{\prime} \rho(\vec{r}) G_{p}^{(2)}\left(\vec{r}, \vec{r}^{\prime}\right) \rho\left(\vec{r}^{\prime}\right),
$$

where $G_{p}^{(2)}\left(\vec{r}, \vec{r}^{\prime}\right)$ is the two-dimensional Green's function on the conical twogeometry. 
The analogue magnetic expression for the linear energy density can be writen for the particular configuration where the constant current is also paralell to the topological defect. For this situation we have

$$
\frac{U_{M a g}}{\ell}=\frac{1}{2 c^{2}} \iint d^{2} r d^{2} r^{\prime}\left(\vec{J}(\vec{r}) \cdot \vec{J}\left(\vec{r}^{\prime}\right)\right) G_{p}^{(2)}\left(\vec{r}, \vec{r}^{\prime}\right) .
$$

(In the derivation of (5), we assumed that the vector potential $\vec{A}$ associated with the magnetic field $\vec{B}$ is in the Coulomb gauge; i. e., $\vec{\nabla} \cdot \vec{A}=0$ ).

Now, by using equations (4) and (5), we obtain the linear self-energy densities, admiting for the electric and magnetic sources the expressions below:

$$
\rho(\vec{r})=\lambda \frac{\delta\left(r-r_{0}\right) \delta\left(\theta-\theta_{0}\right)}{r}=\lambda \delta^{(2)}\left(\vec{r}-\vec{r}_{0}\right)
$$

and

$$
\vec{J}(\vec{r})=j_{0} \hat{z} \frac{\delta\left(r-r_{0}\right) \delta\left(\theta-\theta_{0}\right)}{r}=j_{0} \hat{z} \delta^{(2)}\left(\vec{r}-\vec{r}_{0}\right),
$$

which correspond to the physical situations that we have explained above.

Substituting (6) into (4), and (7) into (5), we get the following linear self-energy densities

$$
\frac{U_{\text {Ele }}}{\ell}=1 /\left.2 \lambda^{2} G_{p}^{(2)}\left(\vec{r}_{0}, \vec{r}_{0}\right)\right|_{\text {Ren }}
$$

and

$$
\frac{U_{M a g}}{\ell}=\left.\frac{j_{0}^{2}}{2 c^{2}} G_{p}^{(2)}\left(\vec{r}_{0}, \vec{r}_{0}\right)\right|_{\text {Ren }} .
$$

In order to obtain a finite result for the linear self-energy densities given above, in the evaluation of the Green's function we adopt a renormalization procedure to extract its irregular part, as indicated in our notation. On the other hand, we have to be sure that no self-energy survives in the absence of the topological defect. So, we subtract from the Green's function 
its Minkowski space-time correspondent and only then take the coincidence limit. In this way, we have

$$
\left.G_{p}^{2}\left(\vec{r}_{0}, \vec{r}_{0}\right)\right|_{\text {Ren }}=\lim _{\vec{r} \rightarrow \vec{r}_{0}}\left[G_{p}^{(2)}\left(\vec{r}, \vec{r}_{0}\right)-G_{1}^{(2)}\left(\vec{r}, \vec{r}_{0}\right)\right] .
$$

As we shall see, this procedure gives us a well-defined expression for equations (8) and (9). Now, all that we have to do, is to obtain the conical geometry two-dimensional Green's function, $G_{p}^{(2)}\left(\vec{r}, \vec{r}^{\prime}\right)$, solution of the differential equation

$$
\nabla_{(2)}^{2} G_{p}^{(2)}\left(\vec{r}, \vec{r}^{\prime}\right)=-4 \pi \delta^{(2)}\left(\vec{r}-\vec{r}^{\prime}\right) .
$$

$G_{p}^{(2)}\left(\vec{r}, \vec{r}^{\prime}\right)$, in the angular variables, presents periodicity $2 \pi \alpha$, whereas the two-dimensional Laplacian operator, $\nabla_{(2)}^{2}$, in cylindrical coordinates reads

$$
\nabla_{(2)}^{2}=\frac{1}{r} \partial_{r} r \partial_{r}+\frac{1}{r^{2}} \partial_{\theta}^{2} .
$$

For equation (11), we otained the two-dimensional Green's function

$$
G_{p}^{(2)}\left(\vec{r}, \vec{r}^{\prime}\right)=-\ln \left[r^{2 p}+r^{\prime 2 p}-2\left(r r^{\prime}\right)^{p} \cos p\left(\theta-\theta^{\prime}\right)\right],
$$

which, as we can see, presents in both angular variables a periodicity $2 \pi \alpha$ and reduces to the standard two-dimensional Green's function for the $p=1$ case [10].

At this point, we are in position to obtain the electric and magnetic linear self-energy densities by calculating $\left.G_{p}^{(2)}\left(\vec{r}_{0}, \vec{r}_{0}\right)\right|_{\text {Ren }}$ from $(10)$ :

$$
\left.G_{p}^{(2)}\left(\vec{r}_{0}, \vec{r}_{0}\right)\right|_{\text {Ren }}=-\lim _{\vec{r} \rightarrow \vec{r}_{0}} \ln \left[\frac{r^{2 p}+r_{0}^{2 p}-2\left(r r_{0}\right)^{p} \cos p\left(\theta-\theta_{0}\right)}{r^{2}+r_{0}^{2}-2 r r_{0} \cos \left(\theta-\theta_{0}\right)}\right] .
$$

This limit can be obtained, independently, by two distinct ways:(i) taking $r=r_{0}$ and $\theta \rightarrow \theta_{0}$, or (ii) $\theta=\theta_{0}$ and $r \rightarrow r_{0}$. In both cases we get 


$$
\left.G_{p}^{(2)}\left(\vec{r}_{0}, \vec{r}_{0}\right)\right|_{\text {Ren }}=-2\left[\ln p+(p-1) \ln r_{0}\right],
$$

which is well defined at the point $\vec{r}_{0} \neq 0$ and vanishes for $p=1$; i.e., in the absence of the topological defect.

For the linear self-energy densities we get

$$
\frac{U_{\text {Ele }}}{\ell}=-\lambda^{2}\left[\ln p+(p-1) \ln r_{0}\right]
$$

and

$$
\frac{U_{M a g}}{\ell}=-\frac{j_{0}^{2}}{c^{2}}\left[\ln p+(p-1) \ln r_{0}\right] .
$$

The electrostatic self-force on the linear charge distribution, induced by the lack of flatness of this conical two-geometry, can be obtained by taking the negative gradient of (16). Doing this, we get

$$
\frac{\vec{F}_{E l e}}{\ell}=-\vec{\nabla}\left(\frac{U_{E l e}}{\ell}\right)=\frac{(p-1) \lambda^{2}}{r_{0}} \hat{r}
$$

which is a repulsive force for $p>1$, in agreement with previous results $[3,4]$ for a charged point particle placed in this conical space-time.

The magnetostatic self-force cannot be obtained from the linear selfenergy in this way and, for this reason, we adopt another procedure. (We shall discuss the magnetostatic self-force in an energetic context at the end of this paper.). The magnetostatic forces on an arbitrary distribution of electric current densities, can be obtained by the following expression:

$$
\vec{F}_{M a g}=\frac{1}{c} \int d^{3} r \vec{J}(\vec{r}) \times \vec{B}(\vec{r}),
$$

where the magnetic field $\vec{B}(\vec{r})=\vec{\nabla} \times \vec{A}(\vec{r})$. The vector potential, $\vec{A}(\vec{r})$, for a straight wire carrying a constant current can be expressed, in the Coulomb gauge,by 


$$
\vec{A}(\vec{r})=\frac{1}{c} \int d^{2} r G_{p}^{(2)}\left(\vec{r}, \vec{r}^{\prime}\right) \vec{J}\left(\vec{r}^{\prime}\right) .
$$

Substituting (20) into (19), and after a few steps, we get the magnetic self-force per unit length

$$
\frac{\vec{F}_{M a g}}{\ell}=\frac{1}{c^{2}} \iint d^{2} r d^{2} r^{\prime}\left(\vec{J}(\vec{r}) \cdot \vec{J}\left(\vec{r}^{\prime}\right)\right) \vec{\nabla} G_{p}^{(2)}\left(\vec{r}, \vec{r}^{\prime}\right) .
$$

Finally, the expression for the linear magnetic self-force density can be found by substituting the current density, $\vec{J}(\vec{r})$ given by (7), into (21) yielding

$$
\frac{\vec{F}_{M a g}}{\ell}=\left.\frac{j_{0}^{2}}{c^{2}} \vec{\nabla} G_{p}^{(2)}\left(\vec{r}_{0}, \vec{r}_{0}\right)\right|_{R e n}
$$

Again, in order to obtain a finite well-defined expression for (22) we have, as mentioned in our notation, to adopt a renormalization procedure similar to the previous one:

$$
\left.\vec{\nabla} G_{p}^{(2)}\left(\vec{r}_{0}, \vec{r}_{0}\right)\right|_{\text {Ren }}=\lim _{\vec{r} \rightarrow \vec{r}_{0}} \vec{\nabla}\left[G_{p}^{(2)}\left(\vec{r}, \vec{r}_{0}\right)-G_{1}^{(2)}\left(\vec{r}, \vec{r}_{0}\right)\right] .
$$

In calculating (28), we have to take first the derivative and then the coincidence limit. Once again, the coincidence limit can be taken by two different ways as explained before. In both caes, we get for (28) the same result

$$
\left.\vec{\nabla} G_{p}^{(2)}\left(\vec{r}_{0}, \vec{r}_{0}\right)\right|_{\text {Ren }}=-\frac{(p-1)}{r_{0}} \hat{r} .
$$

Leading, consequently, to

$$
\frac{\vec{F}_{M a g}}{\ell}=-\frac{j_{0}^{2}}{c^{2}} \frac{(p-1)}{r_{0}} \hat{r},
$$

which is attractive for $p>1$. 
A particular point which we decided to investigate is how, from the previous expressions for the electric case, we can obtain the magnetotastic selfforce by a Lorentz transformation. In other words, we want to investigate what two different inertial observers detect as the self-force of a linear charge distribution, when one of them is moving parallel to the distribution with velocity $v=c \beta$ and the other one is at rest. In order to answer this question we should take an appropriate Lorentz transformation: a boost of the charge distribution along the z-direction with coefficients $a_{0}^{0}=-\gamma$ and $a_{3}^{0}=-\beta \gamma$, where $\gamma=\left(1-\beta^{2}\right)^{-1 / 2}$. Taking this transformation, we get for the moving observer, besides an electric charge distribution, an electric current density. Hence, this observer will detect an electric and a magnetic self-force, respectively given by

$$
\frac{\vec{F}_{\text {Elec }}^{\prime}}{\ell}=\frac{(p-1) \lambda^{2}}{\left(1-\beta^{2}\right) r_{0}} \hat{r}
$$

and

$$
\frac{\vec{F}_{M a g}^{\prime}}{\ell}=-\frac{(p-1) \lambda^{2} \beta^{2}}{\left(1-\beta^{2}\right) r_{0}} \hat{r} .
$$

Therefore, the total (Lorentz) self-force detected by this observer is

$$
\frac{\vec{F}_{L}^{\prime}}{\ell}=\frac{(p-1) \lambda^{2}}{r_{0}} \hat{r},
$$

in complete agreement with the previous result given by Eq. (18), when the observer is at rest in the original coordinate frame.

Before we finish this paper we would like to make a few comments about our most important results for the conical two-geometry with positive curvature; i.e., $p>1$ : as we have shown, the induced electrostatic self-force on a linear charge density is repulsive, in contrast with the magnetic self-force on the electric constant current, which is attractive. Although we have obtained 
these results in an analytic way, we could also obtain a similar conclusion using the image method, which is only applied for integer $p$. (See Ref. [4].) We can also understand these self-forces by an energetic analysis: using Eqs.(16) and (17), we can see that, although there may exist an arbitrary energy background, which we did not consider, the linear self-energy density decreases as $r_{0}$ goes to infinity. For this reason, the electrostatic self-force is repulsive. However, for the magnetostatic case, the self-force cannot be expressed as the negative gradient of the self-energy. In fact, we found that the self-force is the positive gradient of the self-energy. This is not a fortuitous coincidence: the explanation lies in the derivation of the conservation of energy for a rigid circuit subjected to a virtual displacement $d \vec{r}$, under the influence of the magnetic forces acting on it, keeping all the currents constant. For this case, taking into account the work performed by external sources against the induced electromotive forces, the change in the magnetic energy is given by $d U=\vec{F} \cdot d \vec{r}$ (this subject is exhaustively discussed in Ref. [11]). Considering these facts in our analysis, and according to Faraday's law, it follows that the magnetostatic self-force is expressed as

$$
\frac{\vec{F}_{M a g}}{\ell}=\vec{\nabla}\left(\frac{U_{M a g}}{\ell}\right)
$$

As we can see, the sign in front of the gradient operator in Eq. (29) is positive, producing the same result obtained before in the explit calculation (see Eq.(25)).

With the calculations that we have done involving the self-force on a constant current parallel to an infinite, straight cosmic string, we complete the analysis of the self-forces on electromagnetic sources initiated by DeWitt and DeWitt [12] in 1964. We believe that the results here presented can be of help in understanding the behavior of a superconducting string [8] in the background space-time of a cosmic string and the electromagnetic properties of disclinated media $[5,9]$. 


\section{Acknowledgements}

This work was partially supported by CNPq and FINEP. We are indebted to the referee for pointing out the references given in [10].

\section{References}

1. A. Vilenkin, Phys. Rev. D23, 852 (1981); W.A. Hiscock, Phys. Rev. D31, 3288 (1985); B. Linet, Gen. Rel. Grav. 17, 1109 (1985).

2. S. Deser and R. Jackiw, Comm. Math. Phys. 118, 495 (1988);

Ph. Gebert and R. Jackiw, Comm. Math. Phys. 124, 229 (1989);

V. B. Bezerra, Phys. Rev. D34, 3288 (1987).

3. B. Linet, Phys. Rev. D33, 1833 (1986).

4. A. G. Smith, in Proceedings of Symposium on The Formation and Evolution of Cosmic Strings, edited by G. W. Gibbons, S. W. Hawking and T. Vachaspati (Cambridge University Press, 1990)

5. Cláudio Furtado and Fernando Moraes, Phys. Lett. A 188, 394 (1994).

6. M. O. Katanaev and I. V. Volovich, Ann. Phys. (NY) 216, 1 (1992).

7. The disclination can be obtained by either removing (positive-curvature disclination), or inserting (negative-curvature disclination) a wedge in the solid.

8. E. Witten, Nucl. Phys. B249, 557 (1989).

9. Cláudio Furtado et al., Phys. Lett. A 195, 90 (1994).

10. The solution for the Green's function in a two-dimensional conical space-time, has also been obtained by T. Souradeep and V. Sahni, Phys. Rev. D46, 1616 (1992) and M.E.X. Guimarães and B. Linet, 
Class. Quantum Grav. 10, 1665 (1993); the apparent disagreament by a constant factor of $2 \pi$ between their solution and ours is due to our use of CGS-Gaussian units whereas they use MKS units. We also point out that a solution of the analogue differential equation with another boundary condition was obtained a long time ago by H. M. Macdonald, Proc. Lond. Math. Soc. 26, 156 (1895).

11. W. K. H. Panovsky and M. Phillips, Classical Electricity and Magnetism (Addison-Wesley Publishing Co., Massachusetts, 1962), 2nd ed. pg. 173.

12. C.M. DeWitt and B.S. DeWitt, Physics (N.Y.) 1, 3 (1964). 